\title{
Stochastic differential equations with singular coefficients on the straight line
}

\author{
Rongrong Tian' ${ }^{1}$ Liang Ding ${ }^{2}$ and Jinlong Wei ${ }^{3^{*}}$ (I)
}

${ }^{\text {*Correspondence: }}$

weijinlong@zuel.edu.cn

${ }^{3}$ School of Statistics and

Mathematics, Zhongnan University

of Economics and Law, 430073,

Wuhan, China

Full list of author information is

available at the end of the article

\section{Springer}

\begin{abstract}
Consider the following stochastic differential equation (SDE):$$
X_{t}=x+\int_{0}^{t} b\left(s, X_{s}\right) d s+\int_{0}^{t} \sigma\left(s, X_{s}\right) d B_{s,} \quad 0 \leq t \leq T, x \in \mathbb{R},
$$

where $\left\{B_{s}\right\}_{0 \leq s \leq T}$ is a 1 -dimensional standard Brownian motion on $[0, T]$. Suppose that $q \in(1, \infty], p \in(1, \infty), b=b_{1}+b_{2}, b_{1} \in L^{q}\left(0, T ; L^{p}(\mathbb{R})\right)$ such that $1 / p+2 / q<1$ and $b_{2}$ is bounded measurable, with $\sigma \in L^{\infty}\left(0, T ; \mathcal{C}_{u}(\mathbb{R})\right)$ there being a real number $\delta>0$ such that $\sigma^{2} \geq \delta$. Then there exists a weak solution to the above equation. Moreover, (i) if $\sigma \in \mathcal{C}\left([0, T] ; \mathcal{C}_{u}(\mathbb{R})\right)$, all weak solutions have the same probability law on 1-dimensional classical Wiener space on $[0, T]$ and there is a density associated with the above SDE; (ii) if $b_{2}=0, p \in[2, \infty)$ and $\sigma \in L^{2}\left(0, T ; \mathcal{C}_{b}^{1 / 2}(\mathbb{R})\right)$, the pathwise uniqueness holds.
\end{abstract}

MSC: $60 \mathrm{H} 10$

Keywords: Weak solution; Strong solution; Existence; Pathwise uniqueness

\section{Introduction and main results}

Consider the following stochastic differential equation (SDE) in $\mathbb{R}^{d}$ :

$$
d X_{t}=b\left(t, X_{t}\right) d t+\sigma\left(t, X_{t}\right) d B_{t}, \quad 0<t \leq T, X_{0}=x \in \mathbb{R}^{d},
$$

where $T>0$ is a given real number, $b:[0, T] \times \mathbb{R}^{d} \longrightarrow \mathbb{R}^{d}, \sigma:[0, T] \times \mathbb{R}^{d} \longrightarrow \mathbb{R}^{d \times k}$ are Borel measurable functions and $\left\{B_{t}\right\}_{0 \leq t \leq T}$ is a $k$-dimensional standard Brownian motion defined on a given stochastic basis $\left(\Omega, \mathcal{F},\left\{\mathcal{F}_{t}\right\}_{0 \leq t \leq T}, \mathbb{P}\right)$.

The fundamental theory for (1.1) is developed mainly by Itô and furnishes a very important tool to construct diffusion process. Under the Lipschitz and linear growing conditions, Itô showed the existence and uniqueness of strong solutions.

Later, the result was sharped by a series of authors on the case of bounded measurable coefficients. In [1], Skorokhod proved that (1.1) had a solution under the condition that $b$ and $\sigma$ are only continuous (also see [2]), and then the problem of the uniqueness of solutions becomes important. When $b$ is bounded measurable, $\sigma$ is bounded continuous and $\sigma \sigma^{\top}$ is strictly elliptic, Strook-Varadhan $[3,4]$ showed the uniqueness in the probability laws. This uniqueness result is then strengthened by Veretennikov [5] for strong

(c) The Author(s) 2020. This article is licensed under a Creative Commons Attribution 4.0 International License, which permits use sharing, adaptation, distribution and reproduction in any medium or format, as long as you give appropriate credit to the original author(s) and the source, provide a link to the Creative Commons licence, and indicate if changes were made. The images or other third party material in this article are included in the article's Creative Commons licence, unless indicated otherwise in a credit line to the material. If material is not included in the article's Creative Commons licence and your intended use is not permitted by statutory regulation or exceeds the permitted use, you will need to obtain permission directly from the copyright holder. To view a copy of this licence, visit http://creativecommons.org/licenses/by/4.0/. 
uniqueness if $b$ is only bounded measurable but $\sigma(t, \cdot)$ is Lipschitz continuous uniformly in $t \in[0, T]$.

When the coefficients are not bounded but only integrable, the existence and uniqueness for solutions is more difficult. A breathtaking work in this direction has been established by Krylov-Röckner [6] for $\sigma=I_{d \times d}$ and

$$
b \in L^{q}\left(0, T ; L^{p}\left(\mathbb{R}^{d} ; \mathbb{R}^{d}\right)\right) \quad \text { with } p, q \in[2, \infty) \text { and } \frac{2}{q}+\frac{d}{p}<1 .
$$

This result was then extended by Fedrizzi-Flandoli [7, 8]. Later, Zhang [9] generalized their results to the non-constant diffusion coefficients: $\sigma(t, \cdot)$ is uniformly continuous uniformly in $t \in[0, T], \sigma \sigma^{\top}$ is uniformly elliptic and $\left|\nabla_{x} \sigma\right| \in L^{q}\left(0, T ; L^{p}\left(\mathbb{R}^{d}\right)\right)$ with $p, q \in(1, \infty)$ and $2 / q+d / p<1$. For more details in this direction, we refer to [10-13]. For some extensions and applications, we refer to [14-18] and the references cited therein.

Since $b$ is only integrable in [6], the non-degenerate assumption on $\sigma \sigma^{T}$ is needed. When the diffusion coefficients are degenerate, we should assume $b$ more regular. When $d=1, b$ and $\sigma$ are time independent, satisfying

$$
|b(x)-b(y)| \leq \varrho(|x-y|), \quad \int_{0+} \frac{1}{\varrho(s)} d s=\infty
$$

and

$$
|\sigma(x)-\sigma(y)|^{2} \leq \rho\left(|x-y|^{2}\right), \quad \int_{0+} \frac{1}{\rho(s)} d s=\infty,
$$

where $\varrho$ is a positive increasing concave function, $\rho$ is positive and increasing, YamadaWatanabe [19] proved the pathwise uniqueness. Recently, Fang-Zhang [20] generalized this result to $d \geq 1$. By assuming that there is a small enough constant $c_{0}$ such that when $|x-y| \leq c_{0}, \varrho(|x-y|)=|x-y| r(|x-y|)$ and $\rho(|x-y|)=|x-y| r(|x-y|)\left(r \in \mathcal{C}^{1}\left(\mathbb{R}_{+}\right)\right)$, they derived the pathwise uniqueness.

Set the space $L^{q}\left(0, T ; L^{p}\left(\mathbb{R}^{d}\right)\right), 2 / q+d / p<1$ by $\mathbb{L}$. Then all above results for (1.1) can be summed by the scheme in Table 1. From the table, we will ask: if $b$ is in class of $\mathbb{L}$ and $\sigma$ is non-degenerate, does there exist a unique weak/strong solution to (1.1) if $\sigma$ is continuous or satisfies (1.4)?

To solve the above question, let us consider (1.1) on the straight line,

$$
d X_{t}=b\left(t, X_{t}\right) d t+\sigma\left(t, X_{t}\right) d B_{t}, \quad 0<t \leq T, X_{0}=x \in \mathbb{R},
$$

where $T>0$ is a given real number, $b:[0, T] \times \mathbb{R} \longrightarrow \mathbb{R}, \sigma:[0, T] \times \mathbb{R} \longrightarrow \mathbb{R}$ are Borel measurable functions. We will give a positive answer for the above question, and initially

Table 1 Strong and weak solutions for SDEs

\begin{tabular}{llll}
\hline$b$ & $\sigma$ & strong solution & weak solution \\
\hline continuous & continuous & & $\exists$ \\
bounded & non-degenerate, continuous & & $\exists$, unique \\
bounded & non-degenerate, Lipschitz & $\exists$, unique & \\
$b \in \mathbb{L}$ & non-degenerate, $|\nabla \sigma| \in \mathbb{L}$ & $\exists$, unique & \\
$(1.3)$ & $(1.4)$ & $\exists$, unique & \\
\hline
\end{tabular}


we use $\mathcal{C}_{b}(\mathbb{R})$ to denote the space consisted of functions which is bounded and continuous on $\mathbb{R}$, and use $\mathcal{C}_{u}(\mathbb{R})$ to denote the space consisted of functions which is bounded and uniformly continuous on $\mathbb{R}$. Our first main result is presented now.

Theorem 1.1 Assume that $q \in(1, \infty]$ and $p \in(1, \infty)$. Let $b=b_{1}+b_{2}$ such that $b_{1} \in$ $L^{q}\left(0, T ; L^{p}(\mathbb{R})\right)$ with $1 / p+2 / q<1$ and $b_{2}$ is bounded measurable. Suppose $\sigma \in L^{\infty}(0, T$; $\left.\mathcal{C}_{u}(\mathbb{R})\right)$ and there is a real number $\delta>0$ such that $\sigma^{2} \geq \delta$.

(i) There is a filtered probability space $\left(\tilde{\Omega}, \tilde{\mathcal{F}},\left\{\tilde{\mathcal{F}}_{t}\right\}_{0 \leq t \leq T}, \tilde{\mathbb{P}}\right)$, two processes $\tilde{X}_{t}$ and $\tilde{B}_{t}$ defined for $t \in[0, T]$ such that $\left\{\tilde{B}_{t}\right\}_{0 \leq t \leq T}$ is a 1-dimensional $\left\{\tilde{\mathcal{F}}_{t}\right\}$-Brownian motion and $\left\{\tilde{X}_{t}\right\}_{0 \leq t \leq T}$ is an $\left\{\tilde{\mathcal{F}}_{t}\right\}$-adapted, continuous, 1-dimensional process for which

$$
\tilde{\mathbb{P}}\left(\int_{0}^{T}\left|b\left(t, \tilde{X}_{t}\right)\right| d t<\infty\right)=1,
$$

and almost surely, for all $t \in[0, T]$,

$$
\tilde{X}_{t}=x+\int_{0}^{t} b\left(s, \tilde{X}_{s}\right) d s+\int_{0}^{t} \sigma\left(s, \tilde{X}_{s}\right) d \tilde{B}_{s} .
$$

(ii) If we suppose further that $b_{2}=0, p \in[2, \infty)$ and $\sigma \in L^{2}\left(0, T ; \mathcal{C}_{b}^{\alpha}(\mathbb{R})\right)$ with $\alpha \geq 1 / 2$, then the pathwise uniqueness holds.

Remark 1.1 (i) If $\sigma$ is time independent, then $\sigma \in \mathcal{C}_{b}^{\alpha}(\mathbb{R})$ with $\alpha \geq 1 / 2$ implies (1.4). But if $b$ is time independent, then $b \in L^{p}(\mathbb{R})$ with $p \geq 2$ does not imply (1.3). Therefore, we develop a new and different existence and uniqueness result to (1.5).

(ii) By using the Sobolev embedding theorem, if $\sigma$ is bounded and $\partial_{x} \sigma \in L^{q}\left(0, T ; L^{p}(\mathbb{R})\right)$, then $\sigma \in L^{q}\left(0, T ; \mathcal{C}_{b}^{1-1 / p}(\mathbb{R})\right.$, thus if $p \geq 2$, it suggests that $\sigma \in L^{2}\left(0, T ; \mathcal{C}_{b}^{1 / 2}(\mathbb{R})\right.$. In this sense, we extend Zhang's result ([9]) for $d=1$.

If $\sigma$ is not Hölder continuous in spatial variable but only uniformly continuous, the uniqueness for weak solutions holds true as well if we suppose further that it is continuous in $t$. It is our second main result.

Theorem 1.2 Let $p, q$ and $b_{1}$ be described in Theorem 1.1. Suppose $b_{2}$ is bounded measurable and $b=b_{1}+b_{2}$. Suppose furthermore that $\sigma \in \mathcal{C}\left([0, T] ; \mathcal{C}_{u}(\mathbb{R})\right)$ and there is a real number $\delta>0$ such that $\sigma^{2} \geq \delta$. Then all weak solutions of (1.5) possess the same probability law on 1-dimensional classical Wiener space $(W([0, T]), \mathcal{B}(W([0, T])))$. If one uses $\mathbb{P}_{x}$ to denote the unique probability law on $(W([0, T]), \mathcal{B}(W([0, T])))$ corresponding to the initial value $x \in \mathbb{R}$. For every $f \in L^{\infty}(\mathbb{R})$, we define

$$
P_{t} f(x):=\mathbb{E}^{\mathbb{P}_{x}} f(w(t)), \quad 0<t \leq T,
$$

where $w(t)$ is the canonical realization of a weak solution $\left\{X_{t}\right\}_{0 \leq t \leq T}$ on Wiener space $(W([0, T]), \mathcal{B}(W([0, T])))$. Then $\left\{P_{t}\right\}_{0 \leq t \leq T}$ has the strong Feller property, i.e. each $P_{t}$ maps a bounded measurable function to a bounded continuous function for every $t>0$. Moreover, $P_{t}$ admits a density $p(t, x, y)$ for almost all $t \in[0, T]$. Besides, for every $s>0$ and every $r \in[1, \infty)$,

$$
\int_{s}^{T} \int_{\mathbb{R}}|p(t, x, y)|^{r} d y d t<\infty
$$


Remark 1.2 (i) For $d \geq 1$, Strook-Varadhan [3, 4] have established a general theory for weak solutions to (1.1) by assuming that $\sigma \sigma^{T}$ is uniformly positive definite, bounded and continuous and $b$ is bounded and Borel measurable. However, Strook-Varadhan's result does not cover Theorem 1.2, since we only suppose $b \in L^{q}\left(0, T ; L^{p}(\mathbb{R})\right)+L^{\infty}([0, T] \times \mathbb{R})$.

(ii) Thanks to [21, Lemma p. 75], the uniqueness in probability law implies the pathwise uniqueness for $d=1$, therefore we obtain the existence and uniqueness for strong solutions.

\section{Proof of Theorem 1.1}

Initially, we state two useful lemmas.

Lemma 2.1 ([6, Theorems 10.2, 10.3] and [8, Lemma 3.4]) Suppose that $p, q \in(1, \infty)$ with $1 / p+2 / q<1, b \in L^{q}\left(0, T ; L^{p}(\mathbb{R})\right), a \in L^{\infty}\left(0, T ; \mathcal{C}_{u}(\mathbb{R})\right)$ and there is a real number $\delta>0$ such that $a \geq \delta$. Let $\lambda>0$ and consider the following Cauchy problem:

$$
\left\{\begin{array}{l}
\partial_{t} u(t, x)+\frac{1}{2} a(t, x) \partial_{x}^{2} u(t, x)+b(t, x) \partial_{x} u(t, x) \\
\quad=\lambda u(t, x)-b(t, x), \quad(t, x) \in(0, T) \times \mathbb{R} \\
u(T, x)=0, \quad x \in \mathbb{R} .
\end{array}\right.
$$

(i) There is a unique solution in $L^{q}\left(0, T ; W^{2, p}(\mathbb{R})\right) \cap W^{1, q}\left(0, T ; L^{p}(\mathbb{R})\right)$.

(ii) For this solution, we also have $u \in \mathcal{C}\left([0, T] ; \mathcal{C}_{b}^{1}(\mathbb{R})\right)$ and as $\lambda \rightarrow \infty$,

$$
\sup _{(t, x) \in[0, T] \times \mathbb{R}}\left|\partial_{x} u(t, x)\right| \rightarrow 0
$$

Remark 2.1 We call $u(t, x)$ a solution to the Cauchy problem (2.1) if it lies in $L^{q}(0, T$; $\left.W_{\text {loc }}^{2,1}(\mathbb{R})\right) \cap W^{1, q}\left(0, T ; L_{\text {loc }}^{1}(\mathbb{R})\right)$ such that for every test function $\varphi \in \mathcal{C}_{0}^{\infty}((0, T] \times \mathbb{R})$, the identity

$$
\begin{aligned}
& \int_{0}^{T} \int_{\mathbb{R}} u(t, x) \partial_{t} \varphi(t, x) d x d t-\frac{1}{2} \int_{0}^{T} \int_{\mathbb{R}} a(t, x) \partial_{x}^{2} u(t, x) \varphi(t, x) d x d t \\
& \quad=\int_{0}^{T} \int_{\mathbb{R}} b(t, x) \partial_{x} u(t, x) \varphi(t, x) d x d t+\int_{0}^{T} \int_{\mathbb{R}}[b(t, x)-\lambda u(t, x)] \varphi(t, x) d x d t
\end{aligned}
$$

holds.

Let $\tilde{B}_{t}$ be a 1-dimensional standard Brownian motion, $\sigma \in L^{\infty}\left(0, T ; \mathcal{C}_{u}(\mathbb{R})\right)$ and $\sigma^{2}(t, x)>$ $\delta>0, b \in L^{1}\left(0, T ; L_{\text {loc }}^{1}(\mathbb{R})\right)$, we define $\mathcal{S}_{b, \sigma}$ a class of $\mathcal{F}_{t}$-adapted continuous stochastic process $\tilde{X}_{t}$ on $[0, T]$ satisfying (1.6) and (1.7).

Lemma $2.2\left(\left[9\right.\right.$, Theorem 2.2]) Suppose $\tilde{X} . \in \mathcal{S}_{b, \sigma}$. Let $p, q \in(1, \infty)$ such that $1 / p+2 / q<1$ and $b, f \in L^{q}\left(0, T ; L^{p}(\mathbb{R})\right)$. Then there is a constant $C>0$, which depends on $p, q, T, b$ and $\sigma$, such that

$$
\mathbb{E} \int_{0}^{T} f\left(t, \tilde{X}_{t}\right) d t \leq C\|f\|_{L^{q}\left(0, T ; L^{p}(\mathbb{R})\right)}
$$


We are now in a position to give the proof details of Theorem 1.1.

(i) When $b$ is bounded measurable, the existence of weak solutions can be found in [22, Theorem 1, p. 87]. According to (2.3), when $b=b_{1}+b_{2}, b_{1} \in L^{q}\left(0, T ; L^{p}(\mathbb{R})\right)$ such that $1 / p+2 / q<1$ and $b_{2}$ is bounded measurable, we can follow the proof calculations of [22, Theorem 1, p. 87] (or see [23, Theorem 4.1]) step by step, so we completed the proof.

(ii) We show the pathwise uniqueness by using Itô-Tanaka's trick (see [24]). Let $\sigma(t, x)$ be given in (1.5) and set $a(t, x)=\sigma^{2}(t, x)$. Consider the Cauchy problem (2.1), by using Lemma 2.1 , there is a unique $u \in L^{q}\left(0, T ; W^{2, p}(\mathbb{R})\right) \cap W^{1, q}\left(0, T ; L^{p}(\mathbb{R})\right)$ solving the Cauchy problem (2.1). Moreover, with the help of $1 / p+2 / q<1, u \in \mathcal{C}\left([0, T] ; \mathcal{C}_{b}^{1}(\mathbb{R})\right)$ and $(2.2)$ is true. Therefore, if $\lambda$ is sufficiently large, then $\left\|\partial_{x} u\right\|_{\mathcal{C}\left([0, T] ; \mathcal{C}_{b}^{0}(\mathbb{R})\right)}<1 / 2$. For this fixed $\lambda$, we define $\Phi(t, x)=x+u(t, x)$, then $\Phi$ forms a non-singular diffeomorphism of class $\mathcal{C}^{1}$ uniformly in $t \in[0, T]$ and

$$
\frac{1}{2}<\left\|\partial_{x} \Phi\right\|_{\mathcal{C}\left([0, T] ; \mathcal{C}_{b}\left(\mathbb{R}^{d}\right)\right)}<\frac{3}{2}, \quad \frac{2}{3}<\left\|\partial_{x} \Psi\right\|_{\mathcal{C}_{\left([0, T] ; \mathcal{C}_{b}\left(\mathbb{R}^{d}\right)\right)}<2,}
$$

where $\Psi(t, x)=\Phi^{-1}(t, x)$.

Let $\left(X_{t}, B_{t}\right)_{0 \leq t \leq T}$ be a weak solution of (1.5). By using Itô's formula (see [6, Theorem 3.7]), we have

$$
\begin{aligned}
d \Phi\left(t, X_{t}\right)= & \partial_{t} u\left(t, X_{t}\right) d t+b\left(t, X_{t}\right) \partial_{x} u\left(t, X_{t}\right) d t+\frac{1}{2} a(t, x) \partial_{x}^{2} u\left(t, X_{t}\right) d t \\
& +\partial_{x} u\left(t, X_{t}\right) \sigma\left(t, X_{t}\right) d B_{t}+b\left(t, X_{t}\right) d t+\sigma\left(t, X_{t}\right) d B_{t} \\
= & \left(\partial_{x} u\left(t, X_{t}\right)+1\right) \sigma\left(t, X_{t}\right) d B_{t}+\lambda u\left(t, X_{t}\right) d t .
\end{aligned}
$$

Denote $Y_{t}=\Phi\left(t, X_{t}\right)=X_{t}+u\left(t, X_{t}\right)$, then

$$
\begin{aligned}
d Y_{t} & =\lambda u\left(t, \Psi\left(t, Y_{t}\right)\right) d t+\left(1+\partial_{x} u\left(t, \Psi\left(t, Y_{t}\right)\right) \sigma\left(t, \Psi\left(t, Y_{t}\right)\right) d B_{t}\right. \\
& =: \tilde{b}\left(t, Y_{t}\right)+\tilde{\sigma}\left(t, Y_{t}\right) d B_{t},
\end{aligned}
$$

with $Y_{0}=y=\Phi(0, x)$. To prove the pathwise uniqueness for (1.5), it is sufficient to show the pathwise uniqueness for (2.5) and vice versa. Now, we show this fact and by a scaling transformation, we only need to concentrate our attention on $T=1$.

For any given $0<\varepsilon<1$, let us introduce for $s \geq 0$ an approximating function

$$
\varphi_{\varepsilon}(s)= \begin{cases}s \log \frac{s}{4 \varepsilon}+\frac{3 \varepsilon}{2}, & s \in[2 \varepsilon, \infty), \\ \frac{s^{2}}{2 \varepsilon}-s \log \frac{s}{\varepsilon}-\frac{\varepsilon}{2} & s \in[\varepsilon, 2 \varepsilon), \\ 0, & s \in[0, \varepsilon) .\end{cases}
$$

It follows that $\varphi_{\varepsilon}(s)$ is nonnegative and twice continuously differentiable, with

$$
\varphi_{\varepsilon}^{\prime}(s)= \begin{cases}\log \frac{s}{4 \varepsilon}+1, & s \in[2 \varepsilon, \infty), \\ \frac{s}{\varepsilon}-\log \frac{s}{\varepsilon}-1 & s \in[\varepsilon, 2 \varepsilon), \\ 0, & s \in[0, \varepsilon),\end{cases}
$$


and

$$
\varphi_{\varepsilon}^{\prime \prime}(s)= \begin{cases}\frac{1}{s}, & s \in[2 \varepsilon, \infty), \\ \frac{1}{\varepsilon}-\frac{1}{s} & s \in[\varepsilon, 2 \varepsilon), \\ 0, & s \in[0, \varepsilon) .\end{cases}
$$

Moreover, $\varphi_{\varepsilon}^{\prime}, \varphi_{\varepsilon}^{\prime \prime}$ are nonnegative, and

$$
\varphi_{\varepsilon}^{\prime}(s) s \leq 2 \varphi_{\varepsilon}(s)+s, \quad \varphi_{\varepsilon}^{\prime \prime}(s) s \leq 1 .
$$

Then we extend $\varphi_{\varepsilon}(s)$ on $(-\infty, \infty)$ symmetrically, so $\varphi_{\varepsilon}(s)=\varphi_{\varepsilon}(|s|)$.

Let $\left(Y_{t}, B_{t}\right)_{0 \leq t \leq T}$ and $\left(\tilde{Y}_{t}, \tilde{B}_{t}\right)_{0 \leq t \leq T}$ be two weak solutions of (2.5) on the same probability space $\left(\Omega, \mathcal{F},\left\{\mathcal{F}_{t}\right\}_{0 \leq t \leq 1}, \mathbb{P}\right)$ with the common initial data such that $B_{t} \equiv \tilde{B}_{t}$. For any positive real number $\zeta>0$, denoting by the stopping time

$$
\tau_{\zeta}=\left\{\begin{array}{l}
\inf \left\{0<t<1 ;\left|Y_{t}-\tilde{Y}_{t}\right|>\zeta\right\} \\
1, \quad \text { if }\left|Y_{t}-\tilde{Y}_{t}\right| \leq \zeta \text { for all } t \in(0,1)
\end{array}\right.
$$

Using Itô's rule to $\varphi_{\varepsilon}$, for every $t \in(0,1)$, it yields

$$
\begin{aligned}
\mathbb{E} \varphi_{\varepsilon}\left(Y_{t \wedge \tau_{\zeta}}-\tilde{Y}_{t \wedge \tau_{\zeta}}\right) & \\
= & \mathbb{E} \int_{0}^{t \wedge \tau_{\zeta}} \varphi_{\varepsilon}^{\prime}\left(Y_{s}-\tilde{Y}_{s}\right)\left[\tilde{b}\left(s, Y_{s}\right)-\tilde{b}\left(s, \tilde{Y}_{s}\right)\right] d s \\
& +\frac{1}{2} \mathbb{E} \int_{0}^{t \wedge \tau_{\zeta}} \varphi_{\varepsilon}^{\prime \prime}\left(Y_{s}-\tilde{Y}_{s}\right)\left[\tilde{\sigma}\left(s, Y_{s}\right)-\tilde{\sigma}\left(s, \tilde{Y}_{s}\right)\right]^{2} d s .
\end{aligned}
$$

By Lemma $2.1, u \in \mathcal{C}\left([0,1] ; \mathcal{C}_{b}^{1}(\mathbb{R})\right)$ and $\partial_{x} u \in L^{q}\left(0,1 ; W^{1, p}(\mathbb{R})\right) \subset L^{q}\left(0,1 ; \mathcal{C}_{b}^{1 / 2}(\mathbb{R})\right)$ (since $p \geq 2)$. Combining the fact $(2.4)$ and $\sigma \in L^{2}\left(0,1 ; \mathcal{C}_{b}^{1 / 2}(\mathbb{R})\right) \cap L^{\infty}\left(0,1 ; \mathcal{C}_{u}(\mathbb{R})\right)$, we conclude that $\tilde{b} \in \mathcal{C}\left([0,1] ; \mathcal{C}_{b}^{1}(\mathbb{R})\right), \tilde{\sigma} \in L^{2}\left(0, T ; \mathcal{C}_{b}^{1 / 2}(\mathbb{R})\right)$. Therefore,

$$
\begin{aligned}
\mathbb{E} \varphi_{\varepsilon}\left(Y_{t \wedge \tau_{\zeta}}-\tilde{Y}_{t \wedge \tau_{\zeta}}\right) \\
\leq C \mathbb{E} \int_{0}^{t \wedge \tau_{\zeta}}\left|\varphi_{\varepsilon}^{\prime}\left(Y_{s}-\tilde{Y}_{s}\right)\right|\left|Y_{s}-\tilde{Y}_{s}\right| d s \\
\quad+C \mathbb{E} \int_{0}^{t \wedge \tau_{\zeta}} \kappa(s) \varphi_{\varepsilon}^{\prime \prime}\left(Y_{s}-\tilde{Y}_{s}\right)\left|Y_{s}-\tilde{Y}_{s}\right| d s,
\end{aligned}
$$

where $\kappa \in L^{1}(0,1)$.

In view of (2.6) and (2.7), from (2.8)

$$
\begin{aligned}
& \mathbb{E} \varphi_{\varepsilon}\left(Y_{t \wedge \tau_{\zeta}}-\tilde{Y}_{t \wedge \tau_{\zeta}}\right) \\
& \quad \leq C \mathbb{E} \int_{0}^{t \wedge \tau_{\zeta}} \varphi_{\varepsilon}\left(Y_{s}-\tilde{Y}_{s}\right) d s+C \mathbb{E} \int_{0}^{t \wedge \tau_{\zeta}}\left|Y_{s}-\tilde{Y}_{s}\right| d s+C \mathbb{E} \int_{0}^{t \wedge \tau_{\zeta}} \kappa(s) d s \\
& \quad \leq C \mathbb{E} \int_{0}^{t \wedge \tau_{\zeta}} \varphi_{\varepsilon}\left(Y_{s}-\tilde{Y}_{s}\right) d s+C\left[\mathbb{E} \int_{0}^{t}\left|Y_{s}-\tilde{Y}_{s}\right| d s+1\right] .
\end{aligned}
$$


On the other hand, $Y_{s}$ and $\tilde{Y}_{s}$ are weak solutions of (2.5), and $\tilde{b} \in \mathcal{C}\left([0,1] ; \mathcal{C}_{b}^{1}(\mathbb{R})\right), \tilde{\sigma} \in$ $L^{2}\left(0,1 ; \mathcal{C}_{b}^{1 / 2}(\mathbb{R})\right)$, it can be checked that the last integral in the right hand side of $(2.9)$ is finite uniformly in $t$ on $[0,1]$. Combining Doob's optimal stopping time theorem and a Grönwall type argument, one ends with

$$
\mathbb{E} \varphi_{\varepsilon}\left(Y_{t \wedge \tau_{\zeta}}-\tilde{Y}_{t \wedge \tau_{\zeta}}\right) \leq C
$$

Thanks to Chebyshev's inequality, then

$$
\mathbb{P}\left(\tau_{\zeta} \leq t\right) \varphi_{\varepsilon}(\zeta) \leq \mathbb{E} \varphi_{\varepsilon}\left(Y_{t \wedge \tau_{\zeta}}-\tilde{Y}_{t \wedge \tau_{\zeta}}\right) \leq C
$$

Now, we keep $\zeta>0$ and $t>0$ fixed,

$$
\varphi_{\varepsilon}(\zeta) \rightarrow+\infty, \quad \text { if } \varepsilon \rightarrow 0
$$

so $\mathbb{P}\left(\tau_{\zeta} \leq t\right)=0$ for all $t \in(0,1)$, which implies $\mathbb{P}\left(\tau_{\zeta}<1\right)=0$. By letting $\zeta$ tend to zero, we obtain $\mathbb{P}\left(\tau_{0}<1\right)=0$, i.e. the pathwise uniqueness holds true.

\section{Proof of Theorem 1.2}

Let $\left(X_{t}, B_{t}\right)_{0 \leq t \leq T}$ be a weak solution of $(1.5)$ on a probability space $(\Omega, \mathcal{F}, \mathbb{P})$ with a reference family $\left\{\mathcal{F}_{t}\right\}_{0 \leq t \leq T}$, and let $\left(\tilde{X}_{t}, \tilde{B}_{t}\right)_{0 \leq t \leq T}$ be another weak solution of (1.5) on a probability space $(\tilde{\Omega}, \tilde{\mathcal{F}}, \tilde{\mathbb{P}})$ with a reference family $\left\{\tilde{\mathcal{F}}_{t}\right\}_{0 \leq t \leq T}$. We denote the probability laws of $\left\{X_{t}\right\}_{0 \leq t \leq T}$ and $\left\{\tilde{X}_{t}\right\}_{0 \leq t \leq T}$ on 1-dimensional classical Wiener space $(W([0, T]), \mathcal{B}(W([0, T])))$ by $\mathbb{P}_{x}=\mathbb{P} \circ X^{-1}$ and $\tilde{\mathbb{P}}_{x}=\mathbb{P} \circ \tilde{X}^{-1}$, respectively.

Lemma 3.1 ([2, Corollary, p. 206]) $\mathbb{P}_{x}=\tilde{\mathbb{P}}_{x}$ is equivalent to

$$
\int_{W([0, T])} f(w(t)) \mathbb{P}_{x}(d w)=\int_{W([0, T])} f(w(t)) \tilde{\mathbb{P}}_{x}(d w),
$$

for every $t \in[0, T]$ and every $f \in \mathcal{C}_{b}(\mathbb{R})$.

Let $\lambda>0$, we consider the following Cauchy problem:

$$
\left\{\begin{array}{l}
\partial_{t} u(t, x)+\frac{1}{2} a(t, x) \partial_{x}^{2} u(t, x)+b_{1}(t, x) \partial_{x} u(t, x) \\
\quad=\lambda u(t, x)-b_{1}(t, x), \quad(t, x) \in(0, T) \times \mathbb{R}, \\
u(T, x)=0, \quad x \in \mathbb{R},
\end{array}\right.
$$

where $a(t, x)=\sigma^{2}(t, x)$. By virtue of Lemma 2.1, there is a unique solution $u$ of (3.2). Moreover, if we define $Y_{t}=\Phi\left(t, X_{t}\right)=X_{t}+u\left(t, X_{t}\right), \Psi=\Phi^{-1}$, then (2.4) is true. In view of Itô's rule and using the same notation as in (2.5), it yields

$$
\begin{aligned}
d Y_{t}= & \lambda u\left(t, \Psi\left(t, Y_{t}\right)\right) d t+b_{2}\left(t, \Psi\left(t, Y_{t}\right)\right) \\
& +\left(1+\partial_{x} u\left(t, \Psi\left(t, Y_{t}\right)\right) \sigma\left(t, \Psi\left(t, Y_{t}\right)\right) d B_{t}\right. \\
= & \bar{b}\left(t, Y_{t}\right)+\tilde{\sigma}\left(t, Y_{t}\right) d B_{t} .
\end{aligned}
$$


Therefore, if $\left(X_{t}, B_{t}\right)_{0 \leq t \leq T}$ is a weak solution of (1.5), then $\left(Y_{t}, B_{t}\right)_{0 \leq t \leq T}$ is a weak solution of (3.3), and vice versa.

Now let $\left(X_{t}, B_{t}\right)_{0 \leq t \leq T}$ and $\left(\tilde{X}_{t}, \tilde{B}_{t}\right)_{0 \leq t \leq T}$ be two weak solutions of (1.5) and the probability laws of $X$ and $\tilde{X}$ on $(W([0, T]), \mathcal{B}(W([0, T])))$ be given by $\mathbb{P}_{x}$ and $\tilde{\mathbb{P}}_{x}$, respectively. Correspondingly, we denote by $\mathbb{P}_{y}$ and $\tilde{\mathbb{P}}_{y}$ the probability laws of $Y$ and $\tilde{Y}$, respectively. Since $Y_{t}=\Phi\left(t, X_{t}\right)$ and $\Phi \in \mathcal{C}\left([0, T] ; \mathcal{C}^{1}(\mathbb{R})\right)$ is a diffeomorphism on $\mathbb{R}$ uniformly for every $t \in[0, T]$, the relationships of $\mathbb{P}_{x}$ and $\mathbb{P}_{y}, \tilde{\mathbb{P}}_{x}$ and $\tilde{\mathbb{P}}_{y}$ are given by $\mathbb{P}_{y}=\mathbb{P}_{x} \circ \Psi, \tilde{\mathbb{P}}_{y}=\tilde{\mathbb{P}}_{x} \circ \Psi$. In (3.2), $\bar{b}$ is a bounded measure in $(t, x), \tilde{\sigma}$ is bounded uniformly continuous in $(t, x)$, from [3, Theorem 5.6] (also see [2, Theorem 3.3, p185] for time independent $\sigma$ ), the conclusions for Theorem 1.2 are true for SDE (3.3). On the other hand, $X_{t}=\Psi\left(t, Y_{t}\right)$ and (2.4) is true, and we check that, for every $f \in \mathcal{C}_{b}(\mathbb{R})$ and every $t \in[0, T]$,

$$
\begin{aligned}
\int_{W([0, T])} f(w(t)) \mathbb{P}_{x}(d w) & =\int_{W([0, T])} f(\Psi(t, w(t))) \mathbb{P}_{y}(d w), \\
& =\int_{W([0, T])} f(\Psi(t, w(t))) \tilde{\mathbb{P}}_{y}(d w) \\
& =\int_{W([0, T])} f(w(t)) \tilde{\mathbb{P}}_{x}(d w) .
\end{aligned}
$$

With the help of Lemma 3.1 and by (3.4), the weak solution for SDE (1.5) is unique. Moreover, if we define $P_{t}$ by (1.8), for every bounded measurable function $f$, then

$$
P_{t} f(x)=\int_{W([0, T])} f(w(t)) \mathbb{P}_{x}(d w)=\int_{W([0, T])} f(\Psi(t, w(t))) \mathbb{P}_{y}(d w) .
$$

with $y=\Phi(0, x)$. So, $\left\{P_{t}\right\}_{0 \leq t \leq T}$ possesses the strong Feller property. Besides, $P_{t}$ admits a density $p(t, x, y)$ for almost all $t \in[0, T]$, and if one sets the density for $\operatorname{SDE}(3.3)$ by $\tilde{p}(t, x, y)$, then $p(t, x, y)=\tilde{p}(t, \Phi(0, x), \Phi(t, y))|\nabla \Phi(t, y)|$. Hence (1.9) is true and we finish the proof.

\section{Acknowledgements}

The authors are grateful to the anonymous referees for helpful comments and suggestions, which greatly improved the presentation of this paper.

\section{Funding}

The first author was partially supported by the National Natural Science Foundation of China (No. 11901442). The second author was supported by the research funding project of Guizhou Minzu University (No. GZMU[2019]QN04). The third author was partially supported by the National Natural Science Foundation of China (No. 11501577).

Availability of data and materials

Data sharing not applicable to this article as no datasets were generated or analyzed during the current study.

Competing interests

The authors declare that they have no competing interests.

Authors' contributions

The authors contributed equally to this paper. All authors read and approved the final manuscript.

\section{Author details}

${ }^{1}$ College of Science, Wuhan University of Technology, 430070, Wuhan, China. ${ }^{2}$ School of Data Science and Information Engineering, Guizhou Minzu University, 550025, Guiyang, China. ${ }^{3}$ School of Statistics and Mathematics, Zhongnan University of Economics and Law, 430073, Wuhan, China.

\section{Publisher's Note}

Springer Nature remains neutral with regard to jurisdictional claims in published maps and institutional affiliations. 


\section{References}

1. Skorohod, A.V.: Studies in the Theory of Random Processes. Addison-Wesley, Paris (1965)

2. Ikeda, N., Watanabe, S.: Stochastic Differential Equations and Diffusion Processes. North-Holland, Tokyo (1989)

3. Stroock, D.W., Varadhan, S.R.: Diffusion processes with continuous coefficients i. Commun. Pure Appl. Math. 22, 345-400 (1969)

4. Stroock, D.W., Varadhan, S.R.: Diffusion processes with continuous coefficients ii. Commun. Pure Appl. Math. 22 479-530 (1969)

5. Veretennikov, A.J.: On the strong solutions of stochastic differential equations. Theory Probab. Appl. 24, 354-366 (1979)

6. Krylov, N.V., Röckner, M.: Strong solutions of stochastic equations with singular time dependent drift. Probab. Theory Relat. Fields 131, 154-196 (2005)

7. Fedrizzi, E., Flandoli, F.: Pathwise uniqueness and continuous dependence for sdes with non-regular drift. Stochastics 83, 241-257 (2011)

8. Fedrizzi, E., Flandoli, F.: Hölder flow and differentiability for sdes with nonregular drift. Stoch. Anal. Appl. 31, 708-736 (2013)

9. Zhang, X.: Stochastic homeomorphism flows of sdes with singular drifts and Sobolev diffusion coefficients. Electron. J. Probab. 16, 1096-1116 (2011)

10. Attanasio, S: Stochastic flows of diffeomorphisms for one-dimensional sde with discontinuous drift. Electron. Commun. Probab. 15, 213-226 (2010)

11. Jin, P.: Brownian motion with singular time-dependent drift. J. Theor. Probab. 30, 1499-1538 (2017)

12. Tian, R.R., Wei, J.L., Tang, Y.B.: The second-order parabolic pdes with singular coefficients and applications. Stoch. Anal. Appl. 38, 1102-1121 (2020)

13. Zhang, X.: Strong solutions of sdes with singular drift and Sobolev diffusion coefficients. Stoch. Process. Appl. 115 1805-1818 (2005)

14. Mao, X., Marion, G., Renshaw, E.: Environmental Brownian noise suppresses explosions in population dynamics. Stoch Process. Appl. 97, 95-110 (2002)

15. Qiao, H., Wu, J.-L.: Characterizing the path-independence of the Girsanov transformation for non-Lipschitz sdes with jumps. Stat. Probab. Lett. 119, 326-333 (2016)

16. Wu, B., Wu, J.-L.: Characterising the path-independent property of the Girsanov density for degenerated stochastic differential equations. Stat. Probab. Lett. 133, 71-79 (2018)

17. Guo, Z.K.: $P(p \geq 2)$-Strong convergence in averaging principle for multivalued stochastic differential equation with non-Lipschitz coefficients. Adv. Differ. Equ. 386, 1-12 (2017)

18. Tian, R.R., Ding, L., Wei, J.L., Zheng, S.Y.: Hölder estimates of mild solutions for nonlocal spdes. Adv. Differ. Equ. 150 $1-13$ (2019)

19. Yamada, T., Watanabe, S.: On the uniqueness of solutions of stochastic differential equations. J. Math. Kyoto Univ. 11 155-167 (1971)

20. Fang, S., Zhang, T.: A study of a class of stochastic differential equations with non-Lipschitzian coefficients. Probab. Theory Relat. Fields 132, 356-390 (2005)

21. Yamada, T., Tsuchiya, M., Watanabe, S.: Perturbation of drift-type for Lévy process. J. Math. Kyoto Univ. 11, 73-92 (1973)

22. Krylov, N.V.: Controlled Diffusion Processes. Springer, New York (1980)

23. Zhang, $X$ :: Stochastic differential equations with Sobolev drifts and driven by $\alpha$-stable processes. Ann. Inst. Henri Poincaré Probab. Stat. 49, 1057-1079 (2013)

24. Flandoli, F., Gubinelli, M., Priola, E.: Well-posedness of the transport equation by stochastic perturbation. Invent. Math. $180,1-53(2010)$

\section{Submit your manuscript to a SpringerOpen ${ }^{\odot}$ journal and benefit from:}

- Convenient online submission

- Rigorous peer review

- Open access: articles freely available online

- High visibility within the field

- Retaining the copyright to your article

Submit your next manuscript at $>$ springeropen.com 